University of Nebraska - Lincoln

DigitalCommons@University of Nebraska - Lincoln

Roger Kirby Publications

Research Papers in Physics and Astronomy

November 1971

\title{
Even-Parity Lattice Resonances and Anharmonicity in $\mathrm{Kl}$ : Ag+
}

Roger D. Kirby

University of Nebraska-Lincoln, rkirby1@unl.edu

Follow this and additional works at: https://digitalcommons.unl.edu/physics_kirby

Part of the Physics Commons

Kirby, Roger D., "Even-Parity Lattice Resonances and Anharmonicity in KI: Ag+" (1971). Roger Kirby Publications. 31.

https://digitalcommons.unl.edu/physics_kirby/31

This Article is brought to you for free and open access by the Research Papers in Physics and Astronomy at DigitalCommons@University of Nebraska - Lincoln. It has been accepted for inclusion in Roger Kirby Publications by an authorized administrator of DigitalCommons@University of Nebraska - Lincoln. 
were first observed and brought to our attention by Z.J. Kiss, and the initial quantitative investiga- tions were made by P. Asbeck while he was a summer employee at RCA Laboratories.
${ }^{1}$ B. W. Faughnan, Phys. Rev. (to be published).

${ }^{2}$ K. A. Müller, Th. von Waldkirch, W. Berlinger, and

B. W. Faughnan, Solid State Commun. 9, 1097 (1971).

${ }^{3}$ B. W. Faughnan and Z. J. Kiss, IEEE J. Quant. Electron. QE-5, 17 (1969).

${ }^{4}$ B. W. Faughnan and Z. J. Kiss, Phys. Rev. Letters 21,1331 (1968).

${ }^{5}$ T. C. Ensign and S. E. Stokowski, Phys. Rev. B 1 , 2799 (1970).

${ }^{6} \mathrm{~K}$. A. Müller, W. Berlinger, and R. S. Rubin, Phys. Rev. 186, 361 (1969).

${ }^{7}$ See R. N. Tredgold, Space Charge Conduction in Solids.

(Elsevier, Amsterdam, 1966).

${ }^{8}$ B. W. Faughnan and Z. J. Kiss (unpublished).

${ }^{9}$ M. S. Kosman and E. V. Bursian, Dokl. Akad. Nuak SSSR 115, 483 (1957) [Sov. Phys. Doklady 2, 354 (1957)].

${ }^{10} \mathrm{O}$. N. Tufte and P. W. Chapman, Phys. Rev. $\underline{155}$,
796 (1967).

${ }^{11}$ L. C. Walters and R. E. Grace, J. Phys. Chem. Solids 28, 239 (1967); 28, 245 (1967).

${ }^{12}$ See R. C. Bradt and G. S. Ansell, J. Am. Ceram. Soc. 52, $192(1969)$, for a recent analysis with extensive bibliography.

${ }^{13}$ R. Williams, J. Phys. Chem. Solids 26, 399 (1965).

${ }^{14}$ E. M. Pell, J. Appl. Phys. 31, $291(1960)$.

${ }^{15}$ D. L. Staebler and J. Blanc (unpublished).

${ }^{16}$ J. E. Carnes and A. M. Goodman, J. Appl. Phys. 38, 3091 (1967).

${ }^{17}$ M. A. Lampert and P. Mark, Current Injection in Solids (Academic, New York, 1970), p. 189.

${ }^{18}$ A. E. Paladino, J. Am. Ceram. Soc. 43,476 (1965).

${ }^{19}$ A. E. Paladino, L. G. Rubin, and J. S. Waugh, J.

Phys. Chem. Solids 26, 391 (1965).

\title{
Even-Parity Lattice Resonances and Anharmonicity in $\mathbf{K I}: \mathbf{A g}^{+}$ R. D. Kirby* $\dagger$ Department of Physics and Materials Research Laboratory, ${ }^{*}$ University of Illinois, Urbana, Illinois 61801 and Laboratory of Atomic and Solid State Physics, §Comell University, Ithaca, New York 14850 \\ (Received 6 July 1971)
}

\begin{abstract}
We report here the observation of four impurity-induced lattice resonances in $\mathrm{KI}: \mathrm{Ag}^{+}$. In addition to the well-known $T_{1_{u}}$ symmetry resonant mode at $17.3 \mathrm{~cm}^{-1}$, we have observed two even-parity resonant modes of $A_{1 g}$ and $E_{g}$ symmetries at 25 and $16.35 \mathrm{~cm}^{-1}$, respectively, and a $T_{1 u}$ symmetry gap mode at $86.2 \mathrm{~cm}^{-1}$. The two even-parity modes were detected using farinfrared spectroscopic techniques with the sample in an external electric field. The field induces a strong mixing of the modes of opposite parity and results in large field-induced shifts. The $E_{g}$ symmetry resonance has also been seen in the Raman spectrum of $\mathrm{KI}: \mathrm{Ag}^{+}$. Two weak and broad absorption lines at 29.8 and $44.4 \mathrm{~cm}^{-1}$ are attributed to the $E_{g}+T_{1 u}$ and $A_{1 g}+T_{1 u}$ combination bands, respectively. These results are interpreted in terms of a model in which the $T_{1 u}$ symmetry resonant mode is anharmonically coupled to the even-parity resonances.
\end{abstract}

\section{INTRODUCTION}

In recent years there have been a variety of experimental and theoretical investigations of the vibrational properties of substitutional impurities in alkali halides. Thus far, most of the work has been concerned with an understanding of the odd-parity (optically active) local, ${ }^{1-7}$ gap, ${ }^{8,9}$ and resonant ${ }^{10-15}$ modes. However, there can also be impurity-activated resonances with even parity. These modes do not involve any motion of the impurity ion itself, and because of their even parity, will not be optically active in the absence of an external perturbation. To date, even-parity modes have received little attention, apparently because of the difficulties involved in detecting and identifying them.

Both even-parity resonant and gap modes have been observed indirectly as sidebands of the infraredactive $U$-center local mode in $\mathrm{KI}$ and $\mathrm{KBr} .{ }^{1-3}$ The sidebands were made optically active by an anharmonic coupling between the even-parity resonant and gap modes and the odd-parity local mode. In a lattice-dynamical calculation, Gethins et al. ${ }^{16}$ found it necessary to include rather large forceconstant changes between the impurity ion's nearest neighbors and its fourth nearest neighbors (along [100]) to obtain some quantitative agreement with the experimental results of Timusk and Klein. ${ }^{3}$

There is evidence that even-parity resonances have been observed in low-temperature thermal- 
conductivity measurements on doped alkali halides. In $\mathrm{KI}: \mathrm{Ag}^{+},{ }^{17} \mathrm{KBr}: \mathrm{Li}^{+},{ }^{18}$ and $\mathrm{NaCl}: \mathrm{Cu}^{+},{ }^{19}$ low-temperature dips in the thermal conductivity were observed which did not appear at the correct temperatures to be due to the well-known infrared-active resonances in these systems. However, thermal conductivity is basically a broad-band technique and cannot as of yet furnish precise information on the frequencies, linewidths, or symmetries of the observed resonances.

$\mathrm{KI}: \mathrm{Ag}^{+}$is known to have a $T_{1 u}$ symmetry resonant mode at $17.3 \mathrm{~cm}^{-1} \cdot{ }^{10,13}$ We report here the observation of two even-parity resonant modes of $A_{1 g}$ and $E_{g}$ symmetries in $\mathrm{KI}: \mathrm{Ag}^{+}$and offer evidence for an anharmonic coupling between resonances of opposite parity.

In Sec. II, the experimental results of far-infrared-absorption measurements and Raman-scattering measurements on $\mathrm{Ag}^{+}$-doped $\mathrm{KI}$ are reported. These two experimental techniques allow the observation of both odd-parity and even-parity resonances.

In Sec. III, we develop a model in which the $17.3-\mathrm{cm}^{-1} T_{1 u}$ symmetry resonant mode in $\mathrm{KI}: \mathrm{Ag}^{+}$ is anharmonically coupled to the observed evenparity resonances of $A_{1 g}$ and $E_{g}$ symmetries, and in Sec. IV, the predictions of this model are compared with the various experimental results.

In Sec. V of this paper, we speculate on the causes of some of the discrepancies between the model and experiment, and in the Appendix, we consider the effects of a second-order dipole moment on the experimental properties of $\mathrm{KI}: \mathrm{Ag}^{+}$.

A preliminary report of some of these results has been given elsewhere. ${ }^{20}$

\section{EXPERIMENTAL TECHNIQUES AND RESULTS}

A. Far-Infrared Transmission Measurements

The single-crystal pure and doped KI samples studied in this investigation were grown in an argon atmosphere using the Kyropolous technique. Samples were obtained both from the Crystal Growth Facility at Cornell University and from Dr. F. Rosenberger at the Crystal Growth Laboratory of the University of Utah. No differences were seen in the spectra of the samples obtained from the two sources.

The far-infrared transmission measurements on the pure and doped KI samples were made using interferometric spectrometers in conjunction with cooled germanium bolometers. Most of the impurity-induced absorption measurements were made with a modified RIIC FS 720 Michelson interferometer and a Ga-doped germanium bolometer detector operating at $1.2 \mathrm{~K}$. The samples to be studied were mounted on a rotatable sample ring in a separate chamber of the detector cryostat, and were cooled to $4.2 \mathrm{~K}$ by exchange gas coupling to the outer helium bath.
The absorption spectra were obtained by measuring the far-infrared transmission through both the pure and doped KI samples. This technique yields the impurity-induced absorption coefficient.

All of the electric-field-dependence measurements were made with a lamellar interferometer ${ }^{21}$ and a Ge bolometer cooled to $0.4 \mathrm{~K} .{ }^{22,23}$ The samples were immersed in liquid $\mathrm{He}$ at $4.2 \mathrm{~K}$ for these measurements. The experimental techniques involved in making these measurements have been described elsewhere. ${ }^{24}$

Figure 1 shows the $\mathrm{KI}: \mathrm{Ag}^{+}$far-infrared absorption spectrum at $4.2 \mathrm{~K}$ with an instrumental resolution of $0.25 \mathrm{~cm}^{-1}$. This particular sample contained 100-ppm Ag by weight. The spectrum is seen to be complicated, with at least seven $\mathrm{Ag}$-induced absorption lines. The dominant features of the absorption spectrum are the well-known resonant mode at $17.3 \mathrm{~cm}^{-1}$ and the gap mode at $86.2 \mathrm{~cm}^{-1}$. The resonant mode is slightly broadened by the presence of the two naturally occurring $\mathrm{Ag}$ isotopes (107 and $109 \mathrm{amu}$ ) in nearly equal amounts. The isotopic shift of the gap mode has not yet been measured, but is expected to be small. ${ }^{9,25}$ The spectrum in the region of the gap mode was slightly distorted by two relatively weak absorption lines which were present in both pure and doped samples in unequal amounts. These two lines have the same frequencies $\left(86.3\right.$ and $86.9 \mathrm{~cm}^{-1}$ ) and relative intensities as two of the lines observed by Renk in $\mathrm{OH}^{-}$-doped KI. ${ }^{26}$ Becker $^{27}$ has shown that these lines are not due to $\mathrm{OH}^{-}$impurities. Furthermore, we found no evidence of the $69.7-\mathrm{cm}^{-1} \mathrm{OH}^{-}$-induced line observed by him. In any case, these two lines are not due to $\mathrm{Ag}^{+}$, and their effect $(\sim 10 \%)$ on the observed absorption spectrum has been roughly subtracted out. Thus, the solid curve in the gap-mode region of Fig. 1 shows our estimate of the real $\mathrm{Ag}^{*}$-induced absorption line.

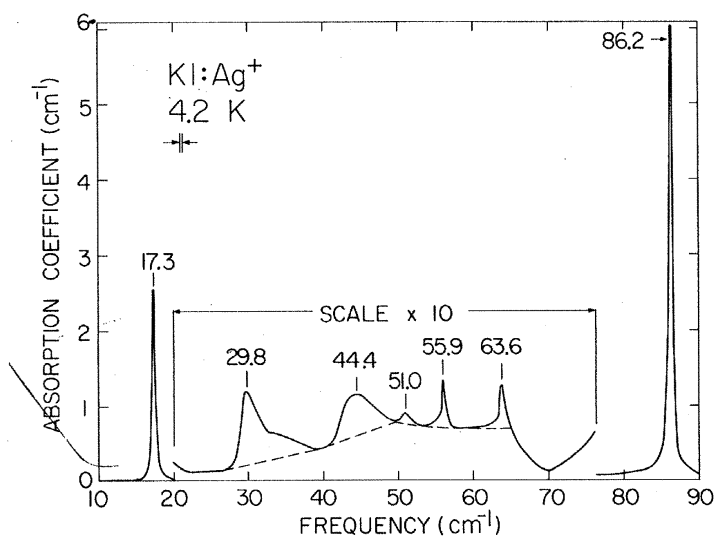

FIG. 1. Far-infrared absorption spectrum of $\mathrm{KI}: \mathrm{Ag}^{+}$ at $4.2 \mathrm{~K}$. The instrumental resolution is $0.25 \mathrm{~cm}^{-1}$. 
TABLE I. Observed far-infrared absorption lines in $\mathrm{KI}: \mathrm{Ag}^{+}$.

\begin{tabular}{ccc}
\hline $\begin{array}{c}\text { Frequency } \\
\left(\mathrm{cm}^{-1}\right)\end{array}$ & $\begin{array}{c}\text { Linewidth } \\
\left(\mathrm{cm}^{-1}\right)\end{array}$ & $\begin{array}{c}\text { Absorption } \\
\text { strength }\end{array}$ \\
\hline 17.3 & 0.60 & 1.0 \\
29.8 & 3.2 & 0.3 \\
44.4 & 6.6 & 0.2 \\
51.0 & 1.6 & 0.01 \\
55.9 & 0.5 & 0.05 \\
63.6 & 0.9 & 0.04 \\
86.2 & 0.65 & 4.0 \\
\hline
\end{tabular}

An absorption peak also occurs at $29.8 \mathrm{~cm}^{-1}$, with a broad shoulder at $\sim 32.8 \mathrm{~cm}^{-1}$. The $44.4-\mathrm{cm}^{-1}$ line is similar to the $29.8-\mathrm{cm}^{-1}$ line in width and strength. The three remaining lines at 51, 55.9, and $63.6 \mathrm{~cm}^{-1}$ are relatively much weaker and narrower.

The observed absorption spectrum varies linearly in absorption strength with Ag concentration, although the $51-\mathrm{cm}^{-1}$ line is too weak to be observed at any but the highest concentrations. The whole absorption spectrum shows the same peculiar aging effect which was reported earlier for the resonant mode. ${ }^{13}$ For example, in one sample, the absorption strength of the resonant mode decreased by a factor of 12 over a period of 15 months. No trace of the $78-\mathrm{cm}^{-1}$ line which was reported to appear after heat treatment ${ }^{13}$ was found in this particular sample. This indicates that more than one type of aging mechanism exists.

The observed $\mathrm{KI}: \mathrm{Ag}^{+}$absorption frequencies, linewidths [full widths at half-maximum (FWHM) absorption ], and integrated absorption strengths (areas under the absorption lines) are summarized in $\mathrm{Ta}-$ ble I. The absorption baselines chosen for the purpose of measuring the linewidths and strengths are indicated in Fig. 1. It should be noted that these baselines are somewhat arbitrary. No corrections for the finite spectrometer resolution have been made, but these corrections are expected to be small. ${ }^{13}$

B. Electric-Field Dependence of the Absorption Spectrum

Figure 2 shows the results of a typical KI: $\mathrm{Ag}^{+}$ electric-field-dependence measurement. An external electric field $\left(E_{\mathrm{dc}}\right)$ of $175 \mathrm{kV} / \mathrm{cm}$ was applied in the [100] crystallographic direction, and the electric-field vector of the incident far-infrared radiation $\left(E_{\mathrm{ir}}\right)$ was polarized parallel to $E_{\mathrm{dc}}$. These data were obtained by dividing the field-on transmission spectrum by the zero-field transmission spectrum and result in the "electric-field-induced absorption coefficient" $(\Delta \alpha)$. In this case, a negative $\Delta \alpha$ indicates a decrease in absorption with applied field, while a positive $\Delta \alpha$ indicates a field-induced in-

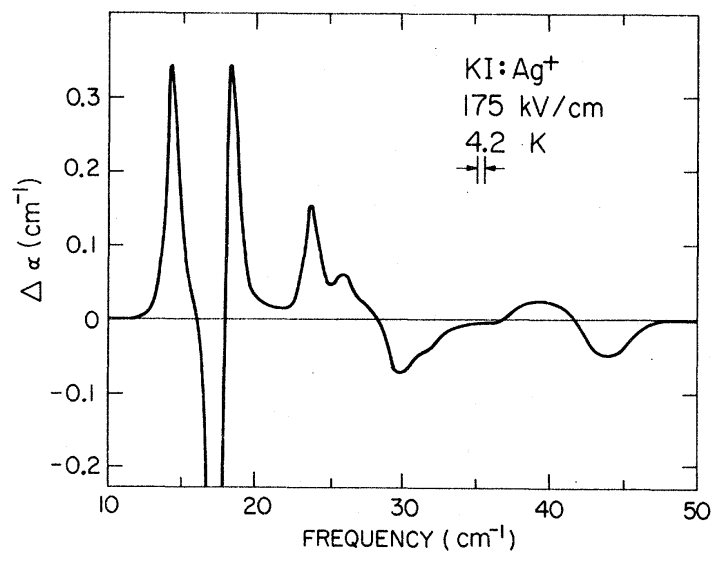

FIG. 2. Electric-field-induced absorption coefficient for KI: $\mathrm{Ag}^{+}$. Here, $E_{\mathrm{ir}}\left\|E_{\mathrm{dc}}\right\|[100]$, and the instrumental resolution is $0.55 \mathrm{~cm}^{-1}$.

crease in absorption at that particular frequency. Clearly, a shift in the absorption frequency of a line will give rise to a negative $\Delta \alpha$ near the unperturbed absorption frequency and a positive $\Delta \alpha$ near the perturbed line position. In the general case, both field-induced dichroisms and shifts are expected to occur.

In the high-field region, much of the field-shifted structure is almost completely resolved from the zero-field structure. In this limit, the zero-field absorption spectrum $[\alpha(0, \nu)]$ can be obtained directly from the negative part of the $\Delta \alpha(E, \nu)$ curve. Then the impurity-induced absorption spectrum with applied field $E$ can be obtained from the relation

$\alpha(E, \nu)=\alpha(0, \nu)+\Delta \alpha(E, \nu)$.

The results of carrying out this procedure for a

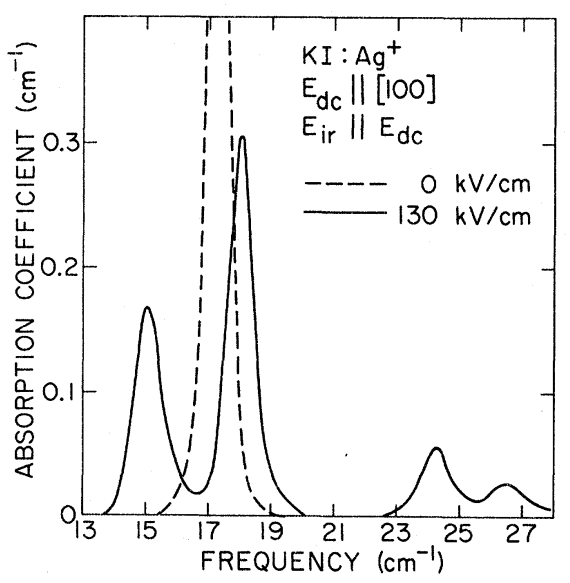

FIG. 3. KI: $\mathrm{Ag}^{+}$absorption spectrum below $20 \mathrm{~cm}^{-1}$ with a dc electric field of $130 \mathrm{kV} / \mathrm{cm}$ applied along [100]. Here, $E_{\mathrm{ir}} \| E_{\mathrm{dc}}$, and the instrumental resolution is 0.35 $\mathrm{cm}^{-1}$. 


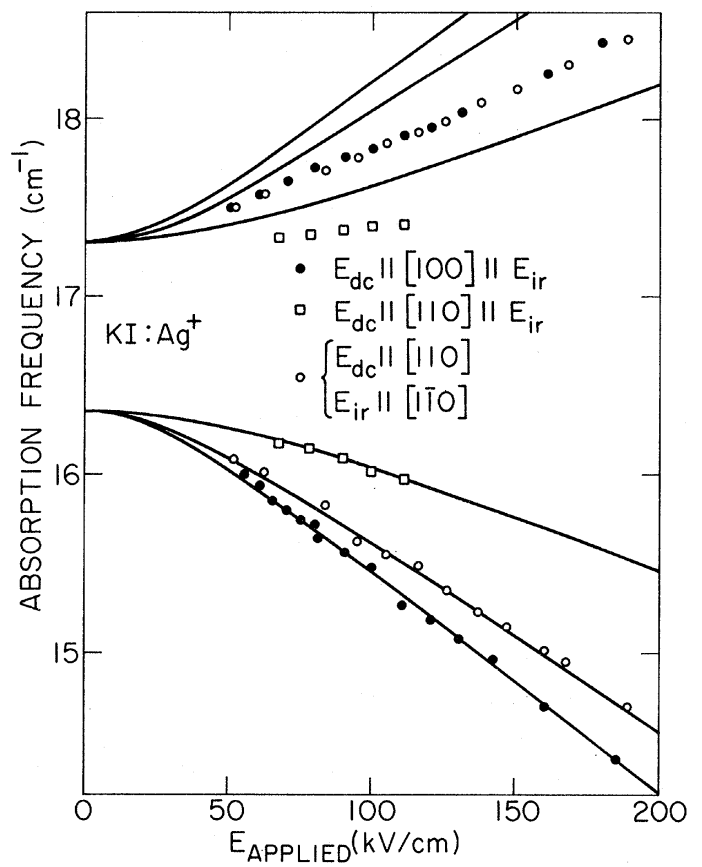

FIG. 4. Frequency vs external electric-field results for the $T_{1 u}$ and $E_{g}$ symmetry resonances in $\mathrm{KI}: \mathrm{Ag}^{+}$. The solid curves were fitted using the anharmonic-oscillator model described in Sec. III.

typical measurement are shown in Fig. 3. Here, $E_{\mathrm{dc}}\|[100]\| E_{\mathrm{ir}}$. The solid curve shows the $\mathrm{KI}: \mathrm{Ag}^{+}$ resonant-mode absorption line in zero field. The dashed curve shows the observed absorption spectrum for $E_{\mathrm{dc}}=130 \mathrm{kV} / \mathrm{cm}$. The field dependence of this spectrum shows that the $18-\mathrm{cm}^{-1}$ line is due to the field-shifted component of the ( $T_{1 u}$ symmetry) resonant mode. It shifts up in frequency and decreases in strength with increasing field. The line near $15.5 \mathrm{~cm}^{-1}$ appears only when a field is applied. It shifts down in frequency and increases in strength with increasing field. The behavior of these two lines is characteristic of two modes of opposite parity being mixed by the (odd-parity) electric field. It will be shown that this is indeed the case and that the lower frequency mode has $E_{g}$ symmetry in zero field.

A somewhat similar field dependence is seen in the $24-30-\mathrm{cm}^{-1}$ region of Fig. 2. One component of the $29.8-\mathrm{cm}^{-1}$ line shifts to lower frequencies and decreases in strength with increasing field. Simultaneously, the $25-\mathrm{cm}^{-1}$ line grows in strength and also shifts down in frequency. We shall show that the $25-\mathrm{cm}^{-1}$ line has $A_{1 \mathrm{~g}}$ symmetry in zero field. However, the mixing in this case is complicated because both lines shift down in frequency. This will be discussed in more detail in Sec. IV.

[100]-field measurements have been made for $E_{\mathrm{ir}} \| E_{\mathrm{dc}}$ and $E_{\mathrm{ir}} \perp E_{\mathrm{dc}}$. For a [110] electric field, there are three distinct polarization directions corresponding to $E_{\mathrm{ir}}\left\|E_{\mathrm{dc}}, E_{\mathrm{ir}}\right\|[1 \overline{1} 0] \perp E_{\mathrm{dc}}$, and $E_{\mathrm{ir}} \|[001] \perp E_{\mathrm{dc}}$. Field-dependence measurements have been made for all three polarization directions. The field-induced shifts for the two lines in the $17-\mathrm{cm}^{-1}$ region are summarized in Fig. 4. For those polarization directions not shown, no shifts were observed. The frequency shifts in all cases are found to be linear with field in the high-field limit. The frequency-vs-field curves for the lower frequency line could not be extended into the lowfrequency region because of its vanishing absorption strength and its proximity to the resonant-mode absorption line. For these reasons the zero-field frequency of this line is known only to an accuracy of $16.35 \pm 0.1 \mathrm{~cm}^{-1}$. The meanings of the solid curves in Fig. 4 will be explained in Sec. IV.

For a [100] field, frequency shifts are observed only for $E_{\mathrm{ir}} \| E_{\mathrm{dc}}$. For a [110] field, no shifts are found with $E_{\mathrm{ir}} \|[001]$. We now use these results to show that the $16.35-\mathrm{cm}^{-1}$ mode does have $E_{g}$ symmetry. With a [100] external field, the defect site symmetry is reduced from $O_{h}$ to $C_{4 v}$, so that an $E_{g}$ mode splits into two levels, transforming as the $A_{1}$ and $B_{1}$ irreducible representations of $C_{4 v}$. Of these, only the $A_{1}$ is optically active, and then only for $E_{\mathrm{ir}} \| E_{\mathrm{dc}}$. For a [110] applied field, the site symmetry becomes $C_{2 v}$ and the $E_{g}$ mode splits into levels

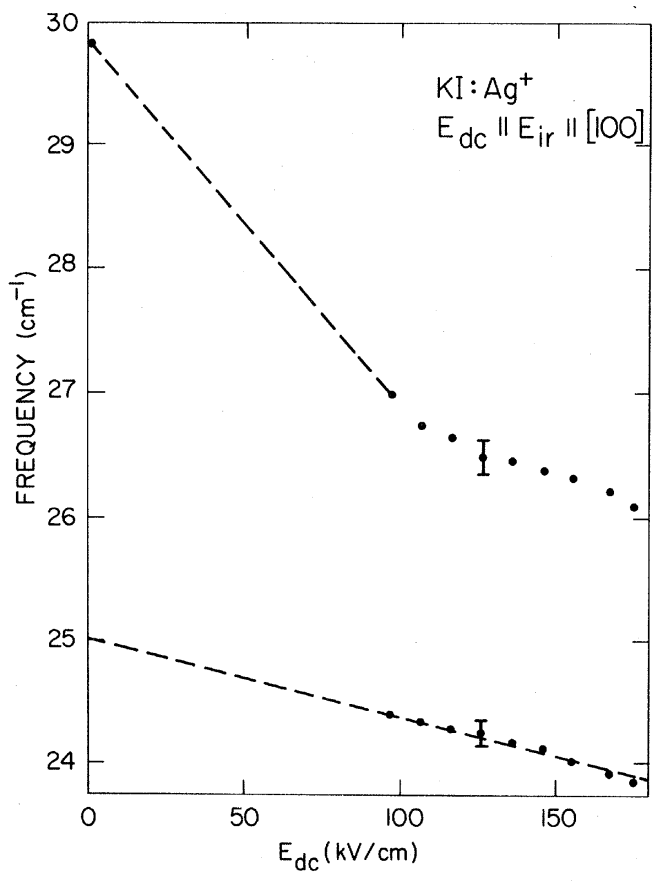

FIG. 5. Frequency vs external electric-field results for the $A_{1 g}$ symmetry resonant mode and the $29.8-\mathrm{cm}^{-1}$ line. Here, $E_{\mathrm{tr}}\left\|E_{\mathrm{dc}}\right\|[100]$. The circles are the experimental data. The curves are explained in the text. 


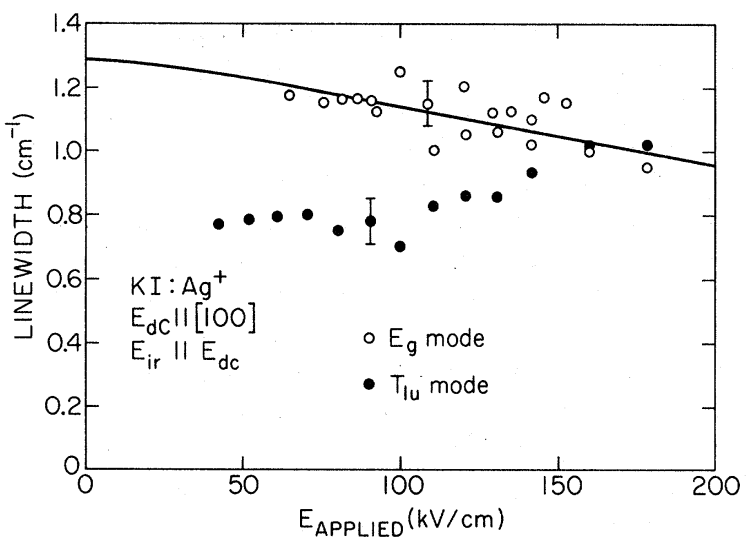

FIG. 6. Linewidth (FWHM absorption) vs external electric-field results for the $T_{1 u}$ and $E_{g}$ symmetry resonances. Here, $E_{\mathrm{ir}}\left\|E_{\mathrm{dc}}\right\|[100]$. No corrections have been made for the instrumental resolution of $0.35 \mathrm{~cm}^{-1}$.

transforming as the $A_{1}$ and $B_{1}$ irreducible representations of $C_{2 v}$. Both of these levels are optically active, the $A_{1}$ for $E_{1 \mathrm{r}} \| E_{\mathrm{dc}}$ and the $B_{1}$ for $E_{\mathrm{ir}} \|$ [110] $\perp E_{\mathrm{dc}}$. This analysis agrees with the experimental results and confirms that the mode in zero field has $E_{g}$ symmetry。

Figure 5 shows the experimental-frequency-vsfield points for the $29.8-\mathrm{cm}^{-1}$ line and the field-induced $25-\mathrm{cm}^{-1}$ line for the $E_{\mathrm{ir}}\left\|E_{\mathrm{dc}}\right\|[100]$. No reliable frequencies could be obtained in the low-field region, but the field dependence of the $29.8-\mathrm{cm}^{-1}$ line below $\sim 100 \mathrm{kV} / \mathrm{cm}$ must be very strong if it is to move up to its zero-field position. This is schematically indicated by the dashed line in Fig. 5 . This may mean that the $25-\mathrm{cm}^{-1}$ line also has a strong low-field dependence so that its zero-field frequency is higher than $25-\mathrm{cm}^{-1}$.

An $A_{1 g}$ mode should be observed with $E_{1 \mathrm{r}} \| E_{\mathrm{dc}}$ for both [100] and [110] fields, but not for $E_{\mathrm{ir}} \perp E_{\mathrm{dc}}$. The $\mathrm{Ag}^{+}$concentrations in the samples used for the $E_{\mathrm{ir}}\left\|E_{\mathrm{dc}}\right\|[110]$ measurements were apparently too low for either the $25-\mathrm{cm}^{-1}$ line or shifts of the 29.8$\mathrm{cm}^{-1}$ line to be observed. It is more significant that the $25-\mathrm{cm}^{-1}$ line was not observed with $E_{\mathrm{dc}} \|$ [110] and $E_{1 \mathrm{r}} \|[1 \overline{1} 0]$ even though shifts of the 29.8$\mathrm{cm}^{-1}$ line were observed. In view of this, we conclude that the even-parity mode near $25 \mathrm{~cm}^{-1}$ does have $A_{1 g}$ symmetry in zero field.

The linewidths (FWHM absorption) exhibit some weak field dependences, as shown in Fig. 6 for $E_{\mathrm{ir}}\left\|E_{\mathrm{dc}}\right\|[100]$. The $T_{1 u}$-mode linewidth (closed circles) increases by $\sim 25 \%$ with increasing field, as does the $A_{1 g}$-mode linewidth (not shown). How ever, the $E_{g}$-mode linewidth (open circles) appears to decrease by a few percent with increasing field. The solid curve has been fit to the $E_{g}$-mode linewidth and will be explained in Sec. III.

\section{Raman Measurements}

Some preliminary Raman spectra of $\mathrm{KI}: \mathrm{Ag}^{+}$were obtained using a Spex double monochrometer and a Coherent Radiation Model 52 argon-ion laser operating with $\sim 1 \mathrm{~W}$ of power at 4880 or $5145 \mathrm{~A}$. Photon-counting techniques were employed to improve the signal-to-noise ratio. The symmetries of any observed scattering peaks could be determined by appropriate combinations of light polarization and crystal orientation. The sample was cooled to $8 \mathrm{~K}$ using a commercially available optical cryostat. During the course of a scan, the sample temperature remained constant to better than $1 \mathrm{~K}$.

Figure 7 shows the 5145-A Raman spectrum of a [110]-cut $\mathrm{KI}: \mathrm{Ag}^{+}$sample for a scattering geometry of $z(y x) y$, which yields scattered light from $E_{\boldsymbol{g}}$ symmetry states. As can be seen, there is a peak at $16.1 \pm 0.5 \mathrm{~cm}^{-1}$, in good agreement with the farinfrared results. The linewidth of $\sim 3 \mathrm{~cm}^{-1}$ is primarily determined by the spectrometer slit width. We were unable to see a Raman peak due to the 25$\mathrm{cm}^{-1} A_{1 g}$ mode, most likely because of the much greater Rayleigh scattering which occurs for that particular scattering configuration.

\section{FORMULATION OF MODEL}

\section{A. Harmonic-Lattice Calculations}

To understand the dynamics of the $\mathrm{KI}: \mathrm{Ag}^{+}$defect-

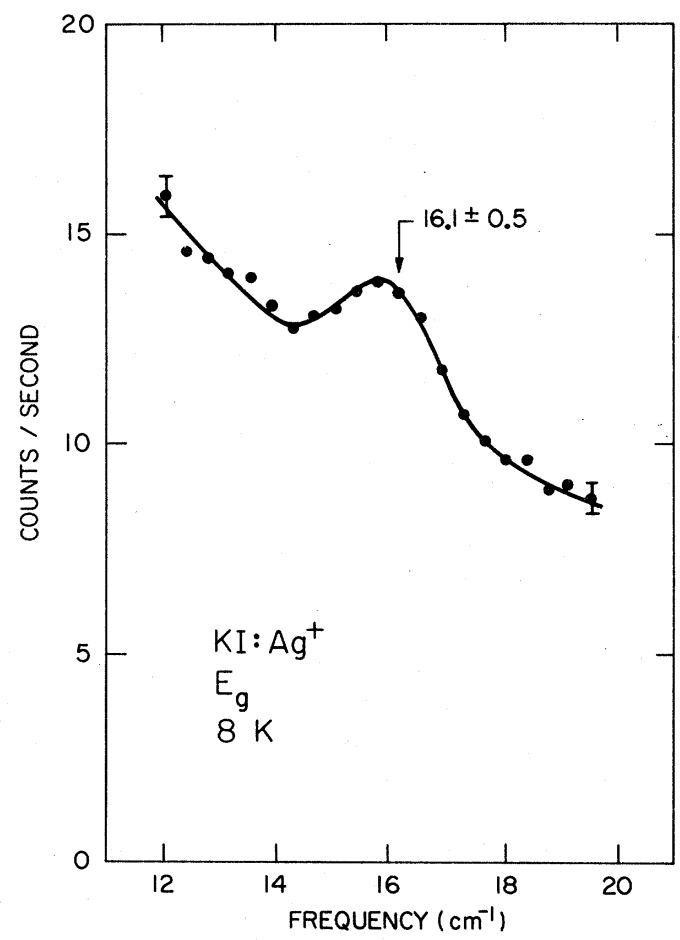

FIG. 7. $E_{g}$ symmetry Raman-scattering spectrum of $\mathrm{KI}: \mathrm{Ag}^{+}$at $8 \mathrm{~K}$. The scattering geometry is $z(y x) y$, with $x\|[110], y\|[110]$, and $z \|[001]$. The instrumental resolution is $2 \mathrm{~cm}^{-1}$. 


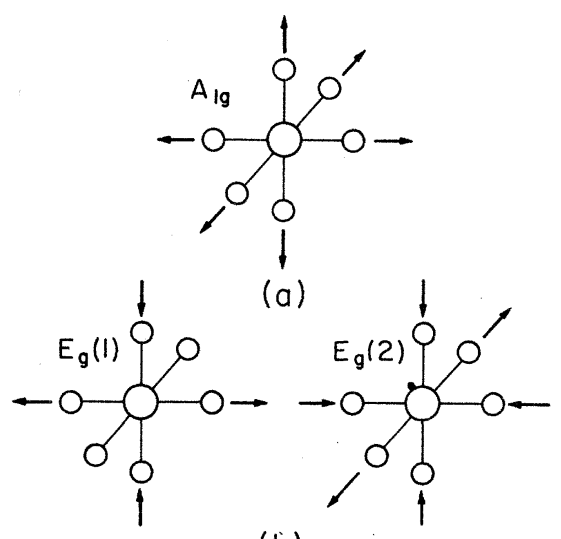

(b)
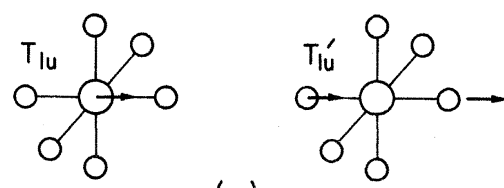

(c)

FIG. 8. Normal modes of an $X Y_{6}$ molecule.

lattice system, we first look to a harmonic nearest-neighbor ( $\mathrm{nn}$ ) model which includes centralforce-constant changes, as has been described by Klein. ${ }^{15}$ It will be seen that such a simplified model cannot explain the experimental results, but it does offer a physical picture of the possible motional states which can cause the observed far-infrared and Raman spectra of $\mathrm{KI}: \mathrm{Ag}^{+}$. In this model, the central displacements of the impurity and its six $\mathrm{nn}$ form a $9 \times 9$ reducible representation of the octahedral group $O_{h}$. This group representation can be reduced by considering the normal modes of the $X Y_{6}$ molecule formed by the impurity and its nn. The $X Y_{6}$ normal modes are found to be an $A_{1 g}$ "breathing" mode, as shown in Fig. 8(a); an $E_{g}$ "tetragonal" mode, both components of which are shown in Fig. 8(b); and two $T_{1 \mathrm{u}}$ modes, with one component of each shown in Fig. 8(c). The two $T_{1 \mathrm{u}}$ modes are normally coupled together when the full lattice-dynamical problem is considered. The modes in Fig. 8 are not true normal modes of the defect-lattice system. They must be considered as linear combinations of the perturbed band modes, which cause a line shape that reflects the systemmode density of states for that particular configuration. The important point here is that the nn-coupling model can lead to two optically active $T_{1 \mathrm{u}}$ resonances (presumably the resonant mode at $17.3 \mathrm{~cm}^{-1}$ and the $86.2-\mathrm{cm}^{-1}$ gap mode) and two even-parity resonances, as we have observed in $\mathrm{KI}: \mathrm{Ag}^{+}$. The addition of noncentral-force-constant changes or the inclusion of a larger defect space only increases the number of possible resonances. Extensions such as these will certainly be necessary, since
Benedek and Nardell ${ }^{12}$ have shown that a nn centralforce-constant model cannot give both a low-lying $T_{1 u}$ resonant mode and a $T_{1 u}$ gap mode in $\mathrm{KI}: \mathrm{Ag}^{*}$. Furthermore, using their calculated $A_{1 g}$ and $E_{g}$ Green's functions for positive impurities in $\mathrm{KI}$, it is easy to show that the $\mathrm{nn}$ effective coupling constant reaches zero long before the even-parity resonances reach such low frequencies, as we have observed.

In addition to the four resonances just discussed, $\mathrm{KI}: \mathrm{Ag}^{+}$shows several other optically active absorption lines which must be explained. The weak and narrow lines at 55.9 and $63.6 \mathrm{~cm}^{-1}$ can be correlated with host-crystal density-of-states peaks, after Dolling et al. ${ }^{28}$ These peaks have been seen in KI doped with other impurities, ${ }^{29,30}$ but they are particularly sharp in the case of $\mathrm{KI}: \mathrm{Ag}^{*}$. The third weak line at $51 \mathrm{~cm}^{-1}$ is not yet understood, but we note here that it is at an appropriate frequency to be associated with the $17.3-\mathrm{cm}^{-1}$ resonant-mode third harmonic.

It is then the two broad lines at 29.8 and 44.4 $\mathrm{cm}^{-1}$ which have yet to be explained and which will be important in the discussion to follow. We will see that these lines are combination bands, corresponding to the simultaneous excitation of one $T_{1 u}$ mode quantum and one even-mode quantum. In the harmonic approximation, the combination bands would not be optically active. However, the strong temperature dependence of the $T_{1 u}$ mode observed by Alexander, Hughes, and Sievers ${ }^{31}$ and the large uniaxial stress shifts of the $T_{1 u}$ mode observed by Nolt and Sievers ${ }^{32}$ show that the defect system is very anharmonic. The question remains as to the nature of the anharmonicity.

The uniaxial stress shifts of the $T_{1 u}$ mode have been interpreted in terms of coupling of the mode to the host-crystal phonons. ${ }^{32,33}$ This would seem to be quite a reasonable assumption. However, Alexander et al. ${ }^{31}$ pointed out that coupling to the phonons could not simultaneously explain the temperature dependence of the $T_{1 u}$ mode and the uniaxialstress shifts. They used the measured-stress coupling coefficients to determine the amount of anharmonic coupling to the phonons, and found that the calculated temperature dependence of the absorption strength was a factor of 100 too small. Furthermore, if they assumed that the $T_{1 u}$ mode was anharmonically coupled to a postulated $E_{g}$ symmetry resonance, they obtained reasonable agreement for both the temperature dependence and the stress dependence of the $T_{1 u}$ mode. We will see in Sec. IV that these intuitive ideas can be at least partially verified using a simple phenomenological model for the defect-lattice system.

At this point, it is worthwhile to discuss another mechanism which may be of importance in the KI: $\mathrm{Ag}^{+}$system. In the case of the $U$-center local mode 
in alkali halides, it appears that higher-order dipole moment effects are responsible for a few percent of the observed phonon sideband structure. 6,7 Klein ${ }^{15}$ has pointed out that second-order dipolemoment terms may be even more important for resonant modes than for local modes. This would seem to be especially true for $\mathrm{KI}: \mathrm{Ag}^{+}$, since $\mathrm{Ag}^{+}$ has a tendency to form covalent bonds, ${ }^{34}$ and this could be important for the charge-transfer mechanism which gives rise to a second-order dipole moment.

As shown in the Appendix, a second-order dipole moment can make the combination bands optically active, and also cause an electric-field-induced mixing of the modes. In fact, the field-induced shifts due to a second-order dipole moment are identical in form to the field-induced shifts produced by an anharmonic coupling of the odd-and even-parity modes. Thus, the measurements presented here cannot distinguish between the two mechanisms. However, to explain the uniaxial-stress dependence and the temperature dependence of the $T_{1 u}$ mode, we have seen that large anharmonic terms are necessary。 So we will proceed, in Sec. III B, with a perturbation treatment of the even-odd anharmonic coupling, realizing that higher-order dipole-moment effects may also be important.

\section{B. Anharmonic-Oscillator Model}

The observation of two $T_{1 u}$ symmetry modes and two even-parity resonances, all of which are quite narrow, makes feasible a phenomenological treatment of the resonances and their interactions. As a first approximation then, we assume that the $T_{1 u}$, $A_{1 g}$, and $E_{g}$ resonances can be treated as independent harmonic oscillators. No evidence that the gap mode participates in the coupled motion has yet been found, so it will not be considered here. Then, neglecting the phonons, the harmonic Hamiltonian may be written

$$
\begin{aligned}
H_{0}=\sum_{i=1}^{3}\left(P_{T i}^{2} / 2 M_{T}\right)+\sum_{i=1}^{3} \frac{1}{2} M_{T} \Omega_{T}^{2} Q_{T i}^{2} \\
+\sum_{i=1}^{2}\left(P_{E i}^{2} / 2 M_{E}\right)+\sum_{i=1}^{2} \frac{1}{2} M_{E} \Omega_{E}^{2} Q_{E i}^{2}+P_{A}^{2} / 2 M_{A} \\
+\frac{1}{2} M_{A} \Omega_{A}^{2} Q_{A}^{2}, \quad(2)
\end{aligned}
$$

where the subscripts $A, E$, and $T$ refer to the $A_{1 g}$, $E_{g}$, and $T_{1 u}$ modes, respectively, the $Q$ 's are the mode normal coordinates, the $M$ 's are the oscillator effective masses, and the $\Omega$ 's are the corresponding resonant frequencies. In the absence of a latticedynamical calculation, the oscillator masses are not known since the amplitudes of vibration may be large far away from the impurity site. For simplicity, we take $M_{T}=M\left(\mathrm{Ag}^{+}\right), M_{A}=M_{E}=M\left(\mathrm{I}^{-}\right)$, The eigenvectors of $H_{0}$ are denoted by $|k l m ; r s ; t\rangle$, with associated eigenvalues $\left(k+l+m+\frac{1}{2}\right) \hbar \Omega_{T}$ $+\left(r+s+\frac{1}{2}\right) \hbar \Omega_{E}+\left(t+\frac{1}{2}\right) \hbar \Omega_{A}$, in an obvious notation. In this approximation, infrared-absorption measurements would lead to a single ( $\delta$-function) line at $\Omega_{T}$, and Raman scattering would give lines of $A_{1 g}$ and $E_{g}$ symmetries at $\Omega_{A}$ and $\Omega_{E}$, respectively.

To include the effects of anharmonicity, we expand the potential energy in powers of the harmonicmode normal coordinates. Because of the inversion symmetry about the defect site, the (odd-parity) $T_{1 u}$-mode coordinates must enter only as even powers. Then the lowest-order terms in the anharmonic potential will be of the forms $Q_{T i}^{2} Q_{E j}$ and $Q_{T i}^{2} Q_{A}$. Static potential terms such as $Q_{E i}^{3}$ will not be considered, nor will coupling between the two even-parity modes be included. These terms may be important in determining the perturbed evenmode energies, but they cannot be responsible for the presence of the combination bands in the infrared absorption spectrum. Neither can they lead to a strong electric-field dependence, because of the large oscillator masses. ${ }^{35,23}$

Then using the transformation properties of the system normal modes, the most general form of the cubic interaction between the $T_{1 u}$ mode and the even-parity modes may be written

$$
\begin{aligned}
H_{A}^{(3)}= & V_{A}\left(Q_{T 1}^{2}+Q_{T 2}^{2}+Q_{T 3}^{2}\right) Q_{A} \\
& +V_{E}\left[\left(2 Q_{T 1}^{2}-Q_{T 2}^{2}-Q_{T 3}^{2}\right) Q_{E 2}+\sqrt{3}\left(Q_{T 2}^{2}-Q_{T 3}^{2}\right) Q_{E 1}\right] .
\end{aligned}
$$

$H_{A}^{(3)}$ will enter in second order of perturbation theory. The quartic terms, which are of the form $Q_{T i}^{2} Q_{\text {even, }}^{2}$ will enter in first order, and so are expected to contribute to the perturbed energies in the same order as $H_{A}^{(3)}$. However, the quartic terms cannot cause the combination bands to be optically active, and they cannot cause a firstorder electric-field-induced mixing of the evenand odd-parity modes. Furthermore, a large quartic anharmonicity would cause quadratic components in the stress-induced frequency shifts of the $T_{1 u}$ resonant mode, whereas the experimental shifts are found to be linear. ${ }^{32}$ For these reasons, we consider only the perturbation $H_{A}^{(3)}$ in what follows. The one-dimensional analog of this perturbation calculation hàs been considered by Hughes, ${ }^{36}$ in a slightly different context.

We treat $H_{A}^{(3)}$ as a perturbation, finding the second-order perturbed energies $\epsilon_{k l m}^{r s t}$, and the firstorder perturbed eigenvectors $\left|\psi_{k l m}^{r s t}\right\rangle$. The perturbed transition energies from the ground state are listed in Table II for some of the lower-energy states.

\section{COMPARISON WITH EXPERIMENT}

\section{A. Zero-Field Absorption Spectrum}

One method of proceeding now would be to fit the 
TABLE II. Perturbed transition energies from the anharmonic-oscillator model, where $U_{E}=V_{E} / 8 M_{T} \Omega_{T}$ $\times\left(\hbar / 2 M_{E} \Omega_{E}\right)^{1 / 2}$ and $U_{A}=V_{A} / 4 M_{T} \Omega_{T}\left(\hbar / 2 M_{A} \Omega_{A}\right)^{1 / 2}$.

\begin{tabular}{cc}
\hline \hline Final state & Perturbed transition energy \\
\hline$|100 ; 00 ; 0\rangle$ & $\hbar \Omega_{T}-\frac{4 U^{2}}{\Omega_{E}}-\frac{4 U_{E}^{2}}{2 \Omega_{T}+\Omega_{E}}-\frac{8 U_{A}^{2}}{\Omega_{A}}-\frac{2 U_{A}^{2}}{2 \Omega_{T}+\Omega_{A}}$ \\
$|000 ; 10 ; 0\rangle$ & $\hbar \Omega_{E}-\frac{3 U_{E}^{2}}{2 \Omega_{T}-\Omega_{E}}-\frac{3 U_{E}^{2}}{2 \Omega_{T}+\Omega_{E}}$ \\
$|000 ; 00 ; 1\rangle$ & $\hbar \Omega_{A}-\frac{3 U_{A}^{2}}{2 \Omega_{T}-\Omega_{A}}-\frac{3 U_{A}^{2}}{2 \Omega_{T}+\Omega_{A}}$ \\
$|100 ; 10 ; 0\rangle$ & $\hbar \Omega_{T}+\hbar \Omega_{A}-\frac{4 U_{E}^{2}}{\Omega_{E}}-\frac{4 U_{E}^{2}}{2 \Omega_{T}+\Omega_{E}}-\frac{8 U_{A}^{2}}{\Omega_{A}}$ \\
$|100 ; 00 ; 1\rangle$ & $-\frac{5 U_{A}^{2}}{2 \Omega_{T}-\Omega_{A}}-\frac{7 U_{A}^{2}}{2 \Omega_{T}+\Omega_{A}}$ \\
& $\hbar \Omega_{T}+\hbar \Omega_{E}-\frac{4 U_{E}^{2}}{\Omega_{E}}-\frac{11 U_{E}^{2}}{2 \Omega_{T}+\Omega_{E}}-\frac{7 U_{E}^{2}}{2 \Omega_{T}-\Omega_{E}}$ \\
& $-\frac{8 U_{A}^{2}}{\Omega_{A}}-\frac{2 U_{A}^{2}}{2 \Omega_{T}+\Omega_{A}}$ \\
\hline \hline
\end{tabular}

perturbed energies of Table II to the observed mode frequencies and so obtain values for the anharmonicity coefficients $V_{A}$ and $V_{E}$ of Eq. (3). This can be done analytically, but it is not very meaningful since many anharmonic terms which may contribute to the mode shifts in the same order as $H_{A}^{(3)}$ have been neglected. In fact, if this calculation is done, we find that $V_{A}$ must be imaginary. While an imaginary coupling coefficient is not necessarily devoid of physical meaning, it most likely indicates that the neglected anharmonic terms are important. We, then conclude that it is more reasonable to determine $V_{A}$ and $V_{E}$ from the strengths of the combination bands for, as pointed out earlier, these quantities are nearly independent of the cubic and quartic terms not included in the perturbation calculation.

For this purpose, we must calculate the electric dipole matrix elements connecting the perturbed ground state $\left|\psi_{000}^{000}\right\rangle$ with the $T_{l u}$ components of the $E_{g}+T_{1 u}$ and $A_{1 g}+T_{1 u}$ combination bands. The $E_{g}+T_{1 u}$ band consists of two three-fold degenerate states, transforming as $E_{g} \times T_{1 u}=T_{2 u}$. The $T_{1 u}$ level will be optically active, and we find the ratio of its integrated absorption strength to that of the $T_{1 u}$ resonant mode to be

$$
\begin{aligned}
S\left(E_{g}+T_{1 u}\right) / S\left(T_{1 u}\right)= & 8 \hbar_{E}^{2}\left(\Omega_{T}+\Omega_{E}\right) / \\
& M_{T}^{2} M_{E} \Omega_{T} \Omega_{E}^{3}\left(2 \Omega_{T}+\Omega_{E}\right)^{2} .
\end{aligned}
$$

The $A_{1 g}+T_{1 u}$ combination band consists only of one $T_{1 u}$ level, and we calculate a similar ratio

$$
\begin{aligned}
& S\left(A_{1 g}+T_{1 u}\right) / S\left(T_{1 u}\right)=2 \hbar V_{A}^{2}\left(\Omega_{T}+\Omega_{A}\right) / \\
& M_{T}^{2} M_{A} \Omega_{T} \Omega_{A}^{3}\left(2 \Omega_{T}+\Omega_{A}\right)^{2} .
\end{aligned}
$$

In calculating these expressions, it is assumed that the perturbed-mode frequencies can be approximated by the harmonic-mode frequencies. Using the values of the ratios given in Table $I$, setting $M_{A}=M_{E}=M\left(\mathrm{I}^{-}\right), M_{T}=M\left(\mathrm{Ag}^{+}\right)$, and assuming that the harmonic frequencies can be replaced by the observed frequencies, we calculate $V_{E}=5.7 \times 10^{11}$ and $V_{A}=1.8 \times 10^{12} \mathrm{erg} / \mathrm{cm}^{3}$. These coefficients are very large, so that the system is very anharmonic. From the expressions for the perturbed energies of Table II, we calculate that coupling coefficients this large lead to anharmonic shifts of the $T_{1 u}$ resonant frequency of $\sim 10 \mathrm{~cm}^{-1}$, so that the anharmonic contributions to the oscillator energies are nearly as large as the harmonic contributions. This means that a perturbation calculation cannot be expected to be quantitatively correct. It can, however, give a semiquantitative indication of the importance of the anharmonic coupling, as we now show.

\section{B. Electric-Field Dependence of the Absorption Spectrum}

The electric-field-dependence results can verify the form of the coupling terms used in the perturbation calculation. The application of an external electric field in the [100] direction introduces the perturbation

$$
H_{E}=-e_{0}^{*} E_{l} Q_{T 1},
$$

where $E_{l}$ is the local electric field at the impurity site, and $e_{0}^{*}$ is the effective charge associated with the odd-parity mode. Since the $E_{g}$ and $T_{1 u}$ (perturbed) modes are nearly degenerate, we let $2 \Delta$ be their zero-field separation and do first-order degenerate perturbation theory with $H_{E}$. This leads to a mixing of the two modes and results in the field-induced shifts (for $E_{1 \mathrm{r}} \| E_{\mathrm{dc}}$ )

$$
W\left(E_{l}\right)= \pm\left(\Delta^{2}+R^{2} E_{l}^{2}\right)^{1 / 2},
$$

where

$$
R=2 e_{0}^{* \hbar} V_{E} /\left(2 \Omega_{T}-\Omega_{E}\right) \Omega_{E} M_{T}\left(M_{T} M_{E} \Omega_{T} \Omega_{E}\right)^{1 / 2} .
$$

The plus and minus signs in Eq. (7) are to be associated with the $T_{1 u}$ and $E_{g}$ modes, respectively. The results of extending this calculation to the other field and polarization directions are summarized in Table III.

The solid curves in Fig. 4 show the calculated shifts of Table III. The value of $R$ (and hence of $V_{E}$ ) has been chosen to fit the [100]-field dependence of the $E_{g}$ mode. The agreement for the other $E_{g}-$ component shifts is quite satisfactory, and so offers evidence that cubic anharmonicity is the dominant mixing mechanism involved. The value $V_{E}=3.7$ $\times 10^{11} \mathrm{erg} / \mathrm{cm}^{3}$ (setting $e_{0}^{*}=|e|$ and making the Lorentz local-field correction) calculated from the fit to the experimental curves compares favorably with the estimate of $V_{E}$ obtained from the strength 
TABLE III. Calculated electric-field shifts of the $E_{g}-$ and $T_{1 u}$-mode components, where $R=2 e_{0}^{*} \hbar V_{E} /\left(2 \Omega_{T}-\Omega_{E}\right)$ $\times \Omega_{E^{M}} M_{T}\left(M_{T} M_{E} \Omega_{T} \Omega_{E}\right)^{1 / 2}$.

\begin{tabular}{lcc}
\hline \hline \multicolumn{2}{l}{ Polarization } & Calculated shifts \\
\hline$E_{\mathrm{dc}}$ & $E_{\mathrm{ir}}$ & \\
{$[100]$} & {$[100]$} & $\pm\left(\Delta^{2}+R^{2} E_{l}^{2}\right)^{1 / 2}$ \\
{$[100]$} & {$[001]$} & 0 \\
{$[110]$} & {$[110]$} & $\pm\left(\Delta^{2}+\frac{1}{4} R^{2} E_{l}^{2}\right)^{1 / 2}$ \\
{$[110]$} & {$[1 \overline{1} 0]$} & $\pm\left(\Delta^{2}+\frac{3}{4} R^{2} E_{l}^{2}\right)^{1 / 2}$ \\
{$[110]$} & {$[001]$} & 0 \\
\hline
\end{tabular}

of the $E_{\mathrm{g}}+T_{1 u}$ combination band.

In Fig. 4 , the experimental shifts of the $T_{1 u}$-mode components are all smaller than the calculated values. This indicates that mixing of the $A_{1 g}$ and $T_{1 u}$ modes should also be considered. The $T_{1 u}$-mode shifts resulting from the $A_{1 g}-T_{1 u}$ mixing are easily estimated to be on the order of $1 \mathrm{~cm}^{-1}$ for $E_{\mathrm{dc}}=200$ $\mathrm{kV} / \mathrm{cm}$. There must however be other contributing factors, such as coupling to the host-crystal phonons, since the $E_{g}$ - and $T_{1 u}$-mode components do not shift equal amounts when $E_{\mathrm{dc}} \|[110]$ and $E_{\mathrm{ir}}$ $\|[1 \overline{1} 0]$. In this particular field configuration, the $A_{1 g}-T_{1 u}$ mixing cannot cause any shifts.

The field dependences of the $A_{1 g}$ resonances and the $29.8-\mathrm{cm}^{-1}$ combination band are more difficult to understand. We consider Fig. 9, which shows a schematic diagram of the perturbed energy levels. Those levels indicated by a solid line have been observed experimentally. Those denoted by dashed lines have not been observed, but are predicted by the anharmonic-oscillator model. The $T_{1 u^{-}}$and $E_{g^{-}}$ mode second harmonics in the $30-35-\mathrm{cm}^{-1}$ region are quite likely responsible for the "anomalous" electric-field shifts of the $29.8-\mathrm{cm}^{-1}$ combination band. The combination band is predicted to be strongly mixed by the electric field with the mode second harmonics, and only weakly mixed with the $25-\mathrm{cm}^{-1} A_{1 g}$ resonance, resulting in a net downward shift. However, the weak mixing between the $A_{1 g}$ even mode and the combination band creates some difficulties of its own. An estimate shows that the $A_{1 g}$ mode should be shifted slightly $u p$ in frequency by an electric field, since the mixing with the 17. $3-\mathrm{cm}^{-1} T_{1 u}$ mode is stronger than that with the 29. $8-\mathrm{cm}^{-1}$ combination band.

Broadening of the absorption lines with field is due to at least two contributions. Nonuniform field broadening would be expected to cause an additional linewidth proportional to the magnitude of the frequency shift of the line. Another source of broadening is due to the changing phonon density of states as the line is shifted up or down in frequency. Since the density of states is proportional to $\omega^{2}$, this con- tribution to the linewidth should also vary as $\omega^{2}$. The solid curve in Fig. 6 is a least-squares fit to these two broadening terms of the form

$$
\Gamma=\alpha\left[\Omega_{E}\left(E_{l}\right)-\Omega_{E}(0)\right]+\beta \Omega_{E}^{2}\left(E_{l}\right) .
$$

The coefficient $\beta$ is found to be $\beta=0.0048 \mathrm{~cm}$. While the data points do show a good deal of scatter, the fit of the curve is evidence that the linewidth of the $E_{g}$ resonance is primarily determined by one-phonon decay.

\section{Uniaxial-Stress Results}

The KI: $\mathrm{Ag}^{+} T_{1 u}$ resonance at $17.3 \mathrm{~cm}^{-1}$ is known to undergo large frequency shifts with an applied uniaxial stress. ${ }^{32}$ We will show here that large stress-induced shifts are consistent with the anharmonic-oscillator model. Using the cluster model described by Alexander et al.,$^{31}$ in which only coupling of the defect to its $\mathrm{nn}$ is assumed to be important, it can be shown that

$$
V_{A}=\sqrt{3} A M_{T} \Omega_{T} / \sqrt{2} \hbar a, \quad V_{E}=\sqrt{3} B M_{T} \Omega_{T} / \hbar a,
$$

where $A$ and $B$ are the $A_{1 g}$ and $E_{g}$ stress coupling

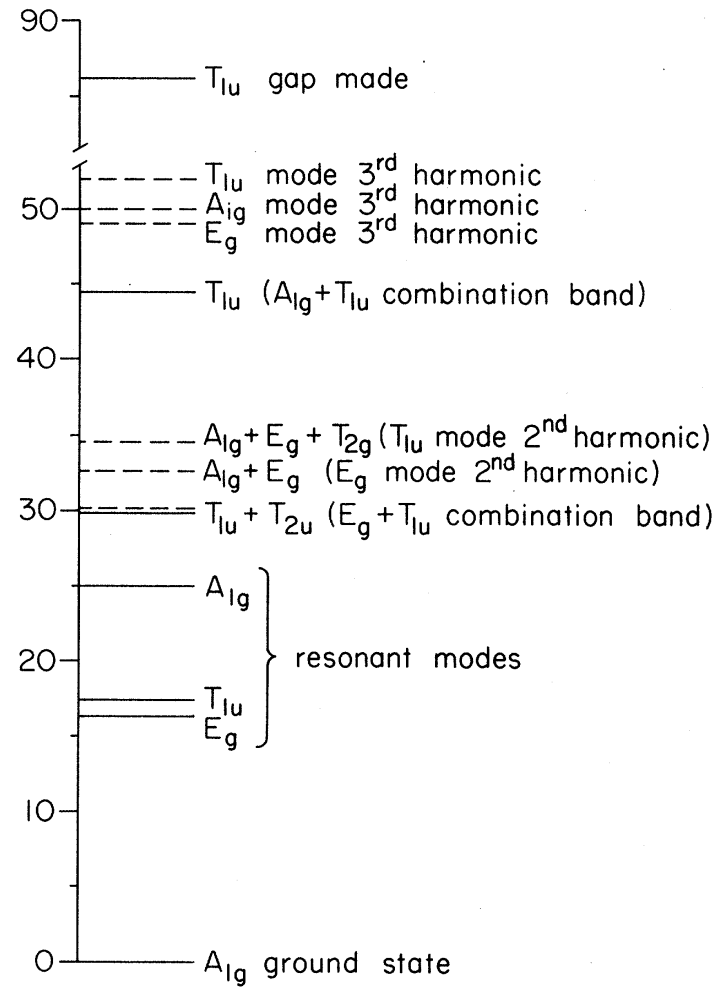

FIG. 9. Schematic diagram of the perturbed vibrational energy levels of $\mathrm{KI}: \mathrm{Ag}^{+}$. Those levels indicated by a solid line have been observed experimentally. Those denoted by a dashed line have not been observed, but are predicted by the anharmonic-oscillator model. Mode harmonics higher than the third are not included in this figure. 
coefficients, ${ }^{32}$ respectively, and $a$ is the nn distance. Using the measured values of $A$ and $B$, corrected for the local compliances at the impurity site, ${ }^{37}$ we calculate $V_{E}=8.8 \times 10^{11}$ and $V_{A}=1.36$ $\times 10^{12} \mathrm{erg} / \mathrm{cm}^{3}$. These values are only in rough agreement with our earlier estimates. This is not too surprising considering the many approximations which have been made. Furthermore, the values of $V_{A}$ and $V_{E}$ obtained from the strengths of the combination bands are a measure of the dynamic coupling of the $T_{1 u}$ mode to the two even modes. The stress measurements on the other hand probe the static coupling. There is no a priori reason why these two coupling coefficients should be the same. Perhaps more important is the fact that the local compliances used in determining the stress coupling coefficients were calculated from a nn centralforce-constant model, ${ }^{33}$ which is clearly not adequate for $\mathrm{KI}: \mathrm{Ag}^{+}$. The important feature here is that the anharmonic-oscillator model can give rise to stress shifts of magnitude similar to those observed.

\section{Temperature of $T_{1 u}$ Mode}

One of the more striking features of the $T_{1 u}$ symmetry resonant modes in alkali halides is their rapid temperature dependence. This is particularly true of $\mathrm{KI}: \mathrm{Ag}^{*}$, where it has been found that the integrated absorption strength of the $T_{1 u}$ mode decreases by a factor of 5 by $15 \mathrm{~K}$. Alexander, Hughes, and Sievers ${ }^{31}$ (hereafter referred to an AHS) showed that this aspect of the temperature dependence could not be explained in terms of linear coupling of the resonant mode to a Debye spectrum of phonons, but some measure of agreement with experiment could be obtained if linear coupling of the $T_{1 u}$ mode to a postulated even-parity resonance of $E_{g}$ symmetry was included. We treat these ideas here using the perturbation approach outlined earlier.

In contrast to linear-coupling theory, as applied by AHS, the perturbation approach does not give a simple analytic solution for the temperature dependence. Here, even a three-dimensional calculation is rather formidable, and to make the problem somewhat more tractable, we resort to a onedimensional calculation in which the $A_{1 g}, E_{g}$, and $T_{1 u}$ modes are treated as if they were singly degenerate. Then the transition energy from a perturbed initial state $\left|n_{T} n_{E} n_{A}\right\rangle$ to a final state $\left|n_{T}+1 ; n_{E} n_{A}\right\rangle$ is given by

$$
\begin{aligned}
\Delta \Omega\left(n_{T}, n_{E}, n_{A}\right) & =\Omega_{T}-\sum_{i=A, E} \frac{V_{i}^{2} \hbar^{2}}{4 M_{T}^{2} \Omega_{T}^{2} M_{i} \Omega_{i}} \\
& \times\left[\frac{4\left(n_{T}+1\right)}{\Omega_{1}}+\frac{n_{T}+2 n_{i}+2}{2 \Omega_{T}+\Omega_{i}}+\frac{2 n_{i}-n_{T}}{2 \Omega_{T}-\Omega_{i}}\right] .
\end{aligned}
$$

The integrated absorption strength of the $T_{1 u}$ mode is then given by

$$
\begin{aligned}
\int_{T_{1 u} \text { mode }} \alpha(\nu) d \nu & \\
=_{n_{T}, n_{E}, n_{A}} & \left\{( 4 \pi ^ { 2 } / 3 c \hbar \hbar n ) \Delta \Omega ( n _ { T } , n _ { E } , n _ { A } ) \left[P\left(n_{T}, n_{E}, n_{A}\right)\right.\right. \\
& \left.\left.\quad-P\left(n_{T}+1, n_{E}, n_{A}\right)\right]\left|\mu\left(n_{T}, n_{E}, n_{A}\right)\right|^{2}\right\}, \quad \text { (10) }
\end{aligned}
$$

where $P\left(n_{T}, n_{E}, n_{A}\right)$ is the Boltzmann population of the state $\left|n_{T} n_{E} n_{A}\right\rangle$, and $\mu\left(n_{T}, n_{E}, n_{A}\right)$ is the electric dipole matrix element connecting $\left|n_{T} n_{E} n_{A}\right\rangle$ with $\left|n_{T}+1 ; n_{E} n_{A}\right\rangle$. To obtain $\mu\left(n_{T}, n_{E}, n_{A}\right)$, the wave functions must be calculated to second order. We have evaluated Eq. (10) by a computer calculation, including all states up to $n_{T}=n_{E}=n_{A}=4$. For temperatures below $15 \mathrm{~K}$, the error incurred in neglecting higher energy states should be negligible. Using the values of the anharmonic coupling coefficients determined from the strengths of the combination bands, the calculation predicts that the integrated absorption strength of the $T_{1 u}$ mode at $15 \mathrm{~K}$ should be $66 \%$ of the low-temperature strength. This should be compared with the experimental results of AHS, who found that the $15-\mathrm{K}$ strength is $20 \%$ of the low-temperature strength.

\section{E. Thermal Conductivity}

Baumann and Pohl $^{17}$ have measured the low-temperature thermal conductivity of $\mathrm{KI}: \mathrm{Ag}^{+}$, finding a dip at $4 \mathrm{~K}$, which indicates the presence of a resonance at $12 \mathrm{~cm}^{-1} \pm 20 \%$. This estimated frequency is somewhat lower than either the $E_{g}$ or $T_{1 u}$ resonances. The $25-\mathrm{cm}^{-1} A_{1 g}$ resonance would be expected to cause a thermal conductivity dip near $8 \mathrm{~K}{ }^{17}$ Experimentally, the $E_{g}$-mode linewidth is found to be a factor of 3 larger than the $T_{1 u}$-mode linewidth. (In making this comparison, it is necessary to use a $T_{1 u}$-mode linewidth of $0.4 \mathrm{~cm}^{-1}$, as has been measured for isotopically pure $\mathrm{Ag}^{+} \mathrm{impu-}$ rities. ${ }^{13}$ The presence of both ${ }^{107} \mathrm{Ag}^{+}$and ${ }^{109} \mathrm{Ag}^{+}$isotopes increases the measured $T_{1 u}$-mode linewidth to $0.6 \mathrm{~cm}^{-1}$, but is expected to have little effect on the even-mode linewidths.) The $E_{g}$-mode linewidth also appears to show the frequency dependence expected for a resonance coupled to the phonons. The greater linewidth of the $E_{g}$ resonance might suggest that it is the more important of the two in determining the low-temperature thermal conductivity. Klein ${ }^{38}$ has recently calculated that the phonon scattering by a resonant mode obeys a "peak-scattering theorem," which says that all resonances of the same degeneracy and frequency in a given host lattice should have the same peak-mean-scattering rate, independent of their widths or shapes. The total scattering strength however varies as (linewidth) ${ }^{2}$. Since the frequencies of the two modes are not very different, one would on this basis ex- 
pect the $E_{g}$ mode to be $\sim 6$ times as effective as the $T_{1 u}$ mode in producing a low-temperature thermal conductivity dip. A possible experimental test of this result is to measure the thermal conductivity of $\mathrm{KI}: \mathrm{Ag}^{+}$with an electric field applied. However, the analyses of such results would be complicated by the nearly complete mixing of the $E_{g}$ and $T_{1 u}$ modes in the high-field region.

\section{SUMMARY AND CONCLUSIONS}

$\mathrm{KI}: \mathrm{Ag}^{+}$shows a complex far-infrared absorption spectrum, with seven optically active energy levels below the TO phonon frequency. We have observed resonant modes of $A_{1 g}, E_{g}$, and $T_{1 u}$ symmetries, as well as a $T_{1 u}$ symmetry gap mode. The presence of four resonant modes with these symmetries is predicted by a nn central-force-constant change model, but the observed resonant frequencies are not consistent with a single force-constant change. Part of the reason for this may be that the system is very anharmonic, but a more plausible explanation is that the defect space used in the lattice-dynamical calculations ${ }^{12}$ was too small. The low frequencies of the even-parity resonance strongly suggest that the lattice-relaxation model of Gethins et al. ${ }^{16}$ which includes force-constant changes between the impurity's nearest neighbors and its fourth nearest neighbors along [100], would be more realistic.

The presence of the $A_{1 g}+T_{1 u}$ and $E_{g}+T_{1 u}$ combination bands and the strong electric-field-induced mixing of the odd- and even-parity resonant modes demonstrates that the two types of resonance are strongly coupled. We have found some agreement between the experimental results on $\mathrm{KI}: \mathrm{Ag}^{+}$and a model in which the $T_{1 u}$ symmetry resonant mode is anharmonically coupled to the observed $A_{1 g}$ and $E_{g}$ symmetry resonances. The perturbation treatment given here explains semiquantitatively the presence of the combination bands and the electric-field shifts of the modes, as well as the uniaxial-stress dependence of the $T_{1 u}$ mode. The agreement for the temperature dependence of the $T_{1 u}$ mode is less satisfactory, but this may be expected in view of the many simplifications used in the calculation.

The question arises as to whether the lack of good agreement for all of the experimental results stems only from the use of an oversimplified model, or if, in addition, there are other mechanisms which are important and have not been taken into account. One purpose of this section is to discuss this aspect of the problem.

The model calculation suffers from several drawbacks. First, the perturbation is rather large. The values of the anharmonic coupling coefficients suggest that the $T_{1 u}$ mode is shifted by $\sim 10 \mathrm{~cm}^{-1}$ by the anharmonicity. Such a large anharmonic contribution to the energy is hardly con- sistent with a perturbation treatment, so that good quantitative agreement cannot be expected.

Second, we have neglected any quartic anharmonicity and any static anharmonicity. Simple arguments tell us that quartic anharmonicity (taken to first order) should be as important as cubic anharmonicity (taken to second order) in determining the perturbed mode energies. Yet, this appears to be inconsistent with a linear shift of the $T_{1 u}$-mode frequency under uniaxial stress.

Third, no coupling of the resonance to the hostcrystal phonons has been included. Such coupling can clearly lead to additional anharmonic shifts of the modes, cause large uniaxial-stress shifts, and explain some features of the temperature dependence. ${ }^{39}$ However, it cannot produce the large observed electric-field shifts or the combination bands, and it cannot explain the temperature dependence of the strength of the $T_{1 u}$ mode.

The combination bands and the electric-field shifts of the modes can be explained by cubic anharmonicity, but these results are equally consistent with the presence of a second-order dipole moment. A second-order dipole moment by itself, however, cannot cause the observed uniaxial-stress shifts and temperature dependence of the $T_{1 u}$ mode. It has been shown ${ }^{40}$ that these two mechanisms are not independent, and furthermore that a large second-order dipole moment implies a large cubic anharmonicity. Thus it appears that both effects will have to be included consistently if we are to understand the $\mathrm{KI}: \mathrm{Ag}^{+}$vibrational spectrum.

While we have not been able to obtain good quantitative agreement with all of the experimental results of $\mathrm{KI}: \mathrm{Ag}^{+}$, the inadequacy of the $\mathrm{nn}$ centralforce-constant change model and the importance of anharmonicity for $\mathrm{KI}: \mathrm{Ag}^{+}$have been demonstrated.

\section{ACKNOWLEDGMENTS}

The author is indebted to Professor M. V. Klien for many helpful discussions and comments on the manuscript, to Professor A. J. Sievers for several stimulating discussions, and to J. G. Peascoe and Dr. G. P. Montgomery for help with the Raman measurements.

\section{APPENDIX: SECOND-ORDER DIPOLE MOMENT EFFECTS}

To include a second-order dipole moment, we start with the harmonic oscillator Hamiltonian [Eq. (2)] and expand the dipole moment $\vec{M}$ in terms of the resonant-mode normal coordinates. If we consider only central motion of the impurity and its neighbors, we may write

$$
\overrightarrow{\mathrm{M}}=\sum_{i=1}^{3} e_{0}^{*} \overrightarrow{\mathrm{Q}}_{T i}+\sum_{i, j=1} a_{i j} Q_{i}^{\mathrm{even}} \overrightarrow{\mathrm{Q}}_{T^{j}}=\overrightarrow{\mathrm{M}}^{(1)}+\overrightarrow{\mathrm{M}}^{(2)},
$$


where $Q_{i}^{\text {even }}$ is one of the even-mode coordinates $Q_{E 1}, Q_{E 2}$, or $Q_{A}, \overrightarrow{\mathrm{M}}^{(1)}$ is the linear dipole moment, and $\vec{M}^{(2)}$ is the second-order dipole moment.

Using group-theoretical arguments, it is easily shown that there are only two independent coefficients $a_{i j}$, so that the $x, y$, and $z$ components of the second-order dipole moment may be written

$$
\begin{aligned}
& M_{x}^{(2)}=2 S_{E} Q_{E 2} Q_{T 1}+S_{A} Q_{A} Q_{T 1}, \\
& M_{y}^{(2)}=-S_{E} Q_{E 2} Q_{T 2}+\sqrt{3} S_{E} Q_{E 1} Q_{T 2}+S_{A} Q_{A} Q_{T 2}, \\
& M_{z}^{(2)}=-S_{E} Q_{E 2} Q_{T 3}-\sqrt{3} S_{E} Q_{E 1} Q_{T 3}+S_{A} Q_{A} Q_{T 3},
\end{aligned}
$$

where $S_{A}$ and $S_{E}$ are constants, and $Q_{E 1}$ and $Q_{E 2}$ are the $E_{g}$-mode normal coordinates, as shown in Fig. 8(b).

The first point to notice is that a second-order dipole moment will make the even-odd combination bands optically active. The ratios of the integrated absorption strengths of the $A_{1 g}+T_{1 u}$ and $E_{g}+T_{1 u}$ combination bands to that of the $T_{1 u}$ resonant mode

$S\left(A_{1 g}+T_{1 u}\right) / S\left(T_{1 u}\right)=\hbar S_{A}^{2}\left(\Omega_{T}+\Omega_{A}\right) / 2 e_{0}^{* 2} M_{A} \Omega_{A} \Omega_{T}$,

$S\left(E_{g}+T_{1 u}\right) / S\left(T_{1 u}\right)=2 \hbar S_{A}^{2}\left(\Omega_{T}+\Omega_{E}\right) / e_{0}^{* 2} M_{E} \Omega_{E} \Omega_{T}$.

Assuming that the observed combination bands are due only to a second-order dipole moment, we calculate $S_{A}=0.2$ and $S_{E}=0.1 \mathrm{statC} / \mathrm{cm}$, using the absorption strengths of Table $I$. These values for the second-order dipole-moment coefficients are an order of magnitude larger than those estimated for pure KI by Xinh. ${ }^{7}$

A second-order dipole moment also leads to electric-field-induced mixing of the $T_{1 u}$ resonance with the even-parity resonances. For simplicity, are found to be

and to facilitate comparison with the cubic anharmonicity calculation, we include here only mixing of the $T_{1 u}$ and $E_{g}$ modes.

Applying a local electric field $\overrightarrow{\mathrm{E}}_{l}$ in the [100] $(x)$ direction reduces the site symmetry from $O_{h}$ to $C_{4 v}$ and introduces the perturbation

$$
H_{S}=-\overrightarrow{\mathrm{M}}^{(2)} \cdot \overrightarrow{\mathrm{E}}_{l}=-M_{x}^{(2)} E_{l}=-2 S_{E} Q_{E 2} Q_{T 1} E_{l} \text {. (A4) }
$$

From first-order degenerate perturbation theory with $H_{S}$, we then obtain secular equation

$$
\begin{gathered}
T_{1 u}(x) \\
T_{1 u}(x) \\
T_{1 u}(y) \\
T_{1 u}(z) \\
E_{g}(1) \\
E_{g}(1)
\end{gathered}\left[\begin{array}{ccccc}
\Delta-W & 0 & 0 & \epsilon E_{l} & 0 \\
0 & \Delta-W & 0 & 0 & 0 \\
0 & 0 & \Delta-W & 0 & 0 \\
\epsilon E_{l} & 0 & 0 & -\Delta-W & 0 \\
0 & 0 & 0 & 0 & -\Delta-W
\end{array}\right]=0,
$$

where $2 \Delta$ is the $E_{g}-T_{1 u}$ zero-field splitting, and

$$
\epsilon=-\hbar S_{E} /\left(M_{T} \Omega_{T} M_{E} \Omega_{E}\right)^{1 / 2} .
$$

Equation (A5) has the solutions

$$
\begin{aligned}
& W\left(A_{1}\right)= \pm\left(\Delta^{2}+\epsilon^{2} E_{l}^{2}\right)^{1 / 2}, \\
& W\left(B_{1}\right)=-\Delta, \quad W(E)=+\Delta,
\end{aligned}
$$

where $A_{1}, B_{1}$, and $E$ are irreducible representations of the $C_{4 v}$ point group.

With a [110] field applied, the site symmetry becomes $C_{2 v}$, and the perturbation is

$$
\begin{aligned}
H_{S}=-2 S_{E} E_{l} Q_{E 2} Q_{T 1} & \left(\frac{1}{2} S_{E} E\right) Q_{E 2} Q_{T 2} \\
& -\frac{1}{2} \sqrt{3} S_{E} E_{l} Q_{E 1} Q_{T 2},
\end{aligned}
$$

which leads to the secular equation

$$
\left[\begin{array}{ccccc}
\Delta-W & 0 & 0 & \epsilon E_{l} / \sqrt{2} & 0 \\
0 & \Delta-W & 0 & -\epsilon E_{l} / 2 \sqrt{2} & \sqrt{3} \epsilon E_{l} / 2 \sqrt{2} \\
0 & 0 & \Delta-W & 0 & 0 \\
\epsilon E_{l} / \sqrt{2} & -\epsilon E_{l} / 2 \sqrt{2} & 0 & -\Delta-W & 0 \\
0 & \sqrt{3} \epsilon E_{l} / 2 \sqrt{2} & 0 & 0 & -\Delta-W
\end{array}\right]=0 .
$$

This has the solutions

$$
\begin{aligned}
& W\left(A_{1}\right)= \pm\left(\Delta^{2}+\frac{1}{4} \epsilon^{2} E_{l}^{2}\right)^{1 / 2}, \\
& W\left(B_{1}\right)= \pm\left(\Delta^{2}+\frac{3}{4} \epsilon^{2} E_{l}^{2}\right)^{1 / 2}, \quad W\left(B_{2}\right)=+\Delta,
\end{aligned}
$$

where $A_{1}, B_{1}$, and $B_{2}$ are irreducible representations of $C_{2 v}$. Assuming that the observed electric- field-induced shifts of the $E_{g}$ mode arise only because of a second-order dipole moment, we obtain $S_{E}=0.15 \mathrm{statC} / \mathrm{cm}$. This is about the same as our estimate of $S_{E}$ from the strength of the combination band.

In view of these results, it must be concluded that one cannot distinguish cubic anharmonicity 
from second-order dipole effects on the basis of electric-field-induced shifts. The relative shifts of the $T_{1 u}$ and $E_{g}$ components are identical for the two mechanisms.
*Present address: Materials Research Laboratory, University of Illinois, Urbana, Ill. 61801.

†Present address: Behlen Laboratory of Physics, Department of Physics, University of Nebraska, Lincoln, Neb. 68508.

*Work supported in part by the Advanced Research Projects Agency under Contract No. HC 15-67-C-0221.

$\S$ Research supported by the U. S. Atomic Energy Commission under Contract No. AT(30-1)-2391. Additional support was received from the Advanced Research Projects Agency through the Materials Science Center at Cornell University.

${ }^{1}$ G. Schaefer, Phys. Chem. Solids 12, 233 (1960).

${ }^{2}$ B. Fritz, U. Gross, and D. Bauerle, Phys. Status Solidi 11,231 (1965).

${ }^{3}$ T. Timusk and M. V. Klein, Phys. Rev. 141, 664 (1966).

${ }^{4}$ S. S. Jaswal and D. J. Montgomery, Phys. Rev. $\underline{138}$, A1257 (1964).

${ }^{5}$ R. Fieschi, G. F. Nardelli, and N. Terzi, Phys. Rev. 138, A203 (1965).

${ }^{6}$ John B. Page, Jr., and B. G. Dick, Phys. Rev. 163, 910 (1967).

${ }^{7}$ Nguyen X. Xinh, Phys. Rev. 163, 896 (1967).

${ }^{8}$ A. J. Sievers, A. A. Maradudin, and S. S. Jaswal, Phys. Rev. 138, A272 (1965).

${ }^{9}$ I. G. Nolt, R. A. Westwig, R. W. Alexander, Jr., and A. J. Sievers, Phys. Rev. 157, 730 (1967).

${ }^{10}$ A. J. Sievers, Phys. Rev. Letters 13, 310 (1964).

${ }^{11} \mathrm{R}$. Weber, Phys. Letters 12 , $311(\overrightarrow{1964})$.

${ }^{12}$ Giorgio Benedek and G. F. Nardelli, J. Chem. Phys. 48,5242 (1967).

${ }^{13}$ R. D. Kirby, I. G. Nolt, R. W. Alexander, Jr., and A. J. Sievers, Phys. Rev. 168, 1057 (1968).

${ }^{14}$ H. F. Macdonald, M. V. Klein, and T. P. Martin, Phys. Rev. 177, 1292 (1969).

${ }^{15}$ For a comprehensive review of the work on impurities in alkali halides through 1967, see the article by M. V. Klein, in Physics of Color Centers, edited by W. B. Fowler (Academic, New York, 1968), Chap. 7.

${ }^{16} \mathrm{~T}$. Gethins, T. Timusk, and E. J. Woll, Phys. Rev. 157, 744 (1967).
17 F. C. Baumann and R. O. Pohl, Phys. Rev. 163, 843 (1967).

${ }^{18}$ F. C. Baumann, J. P. Harrison, R. O. Pohl, and D. W. Seward, Phys. Rev. 159, 691 (1967).

${ }^{19}$ R. F. Caldwell and M. V. Klein, Phys. Rev. 158 , 861 (1967).

${ }^{20}$ R. D. Kirby, Phys. Rev. Letters 26, 512 (1971).

${ }^{21}$ I. G. Nolt, R. D. Kirby, C. D. Lytle, and A. J. Sievers, Appl. Opt. 8, 309 (1969).

${ }^{22}$ H. D. Drew and A. J. Sievers, Appl. Opt. 8, 2067 (1969).

${ }^{23}$ B. P. Clayman, R. D. Kirby, and A. J. Sievers, Phys. Rev. B 3 , 1351 (1971).

${ }^{24}$ R. D. Kirby, A. E. Hughes, and A. J. Sievers, Phys. Rev. B 2, 481 (1970).

${ }^{25}$ B. P. Clayman, I. G. Nolt, and A. J. Sievers, Solid State Commun. 7, 7 (1969).

${ }^{26}$ K. F. Renk, Phys. Letters 20, 137 (1966).

${ }^{27}$ C. R. Becker, Solid State Commun. 8, 337 (1970).

${ }^{28}$ G. Dolling, R. A. Cowley, C. Schittenhelm, and I.

M. Thorson, Phys. Rev. 147, 577 (1966).

${ }^{29}$ A. J. Sievers and C. D. Lytle, Phys. Letters 14, 271 (1965).

${ }^{30}$ K. F. Renk, Phys. Letters 14, 281 (1965).

${ }^{31}$ R. W. Alexander, Jr., A. E. Hughes, and A. J. Sievers, Phys. Rev. B 1,1563 (1970).

${ }^{32}$ I. G. Nolt and A. J. Sievers, Phys. Rev. 174, 1004 (1968).

${ }^{33}$ G. Benedek and G. F. Nardelli, Phys. Rev. 167, 837 (1968).

${ }^{34}$ James C. Phillips, in Covalent Bonding in Crystals, Molecules, and Polymers (Chicago U. P. , Chicago, 1969).

${ }^{35}$ B. P. Clayman and A. J. Sievers, Phys. Rev. Letters 21, 1453 (1968).

${ }^{36}$ A. E. Hughes, Phys. Rev. 173, 860 (1968).

${ }^{37}$ R. J. Elliott, J. A. Krumhansl, and R. H. Merrett, in Localized Excitations in Solids, edited by R. F. Wallis (Plenum, New York, 1968), p. 709.

${ }^{38}$ Miles V. Klein, Phys. Rev. 186, 839 (1969).

${ }^{39}$ K. H. Timmesfeld, Phys. Status Solidi 30, 73 (1968).

${ }^{40}$ B. Szigeti, Proc. Roy. Soc. (London) 252, 217 (1959). 